Western University Scholarship@Western

2018

\title{
Narrowing of Plasmon Resonance Peaks as an Ensemble Effect
}

Zhutian Ding

Western University

James M. Stubbs

Western University

Silvia Mittler

Western University, smittler@uwo.ca

Follow this and additional works at: https://ir.lib.uwo.ca/physicspub

Part of the Astrophysics and Astronomy Commons, and the Physics Commons

Citation of this paper:

Ding, Zhutian; Stubbs, James M.; and Mittler, Silvia, "Narrowing of Plasmon Resonance Peaks as an Ensemble Effect" (2018). Physics and Astronomy Publications. 35.

https://ir.lib.uwo.ca/physicspub/35 
The Journal of Physical Chemistry, in press.

Doi/10.1021/acs.jpcc.8b02301

\section{Narrowing of Plasmon Resonance Peaks as an}

\section{Ensemble Effect}

Zhutian Ding $^{a, b}$, James M. Stubbs ${ }^{b}$, Silvia Mittler ${ }^{*, a, b}$

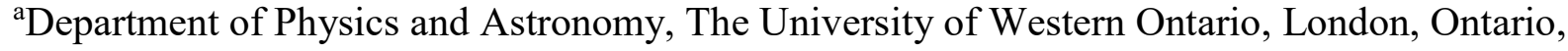
Canada N6A 3 K 7

${ }^{\mathrm{b}}$ Department of Chemistry, The University of Western Ontario, London, Ontario, Canada N6A 5B7 
ABSTRACT: The frequency of localized surface plasmon resonance (LSPR) displayed by gold nanoparticles (AuNPs) redshifts as a function of their local refractive index, which renders them valuable transducers for sensing applications. An ensemble hypothesis is presented herein, along with spectroscopic evidence, using the biotin-streptavidin system on immobilized AuNPs to interpret the decrease in ensemble linewidth (ELW) consistently observed upon functionalization of plasmonic nanoparticles and the subsequent analyte binding. These results demonstrate that ELW can be used to monitor recognition reactions, providing spectral details and a possible sensitivity enhancement to the conventional wavelength sensing. A novel sensing platform allowing the simultaneous measurement of both LSPR wavelength and ELW is proposed, which not only combines the advantages of both parameters but also permits real-time measurement and miniaturization. 


\section{INTRODUCTION}

Gold nanoparticles (AuNPs) exhibit localized surface plasmon resonance (LSPR) whose frequency is dependent on their size, shape, interparticle spacing, and local environment. ${ }^{1-2}$ This latter dependency renders them valuable transducers that convert small changes in the local refractive index into spectral shifts of their extinction spectra. ${ }^{3-4}$

Significant effort has been dedicated to improving the intrinsic sensitivity of LSPR sensors via the optimization of instrumental setups ${ }^{5-6}$; as well as the exploitation of novel geometries ${ }^{7-9}$ and materials ${ }^{10-12}$ for higher polarizabilities. As an alternative to wavelength and intensity sensing, the sensitivity can be further improved extrinsically by monitoring the change in curvature ${ }^{13}$ and inflection points ${ }^{14}$ of the resonance peak. These extrinsic methods are post-measurement manipulations of the extinction spectra that require strategic smoothing algorithms so that the signal to noise ratio $(\mathrm{S} / \mathrm{N})$ is not degraded by numerical differentiation ${ }^{15}$. Thus, challenges exist if one wishes to obtain real-time results on a portable device that relies on a relatively simple and universal processing algorithm.

To detect a specific analyte, AuNPs typically need to be functionalized with a self-assembled monolayer (SAM), with the corresponding recognition moieties exposed on the outside. ${ }^{16-17}$ Spectroscopic measurements ${ }^{18-19}$ and theoretical models ${ }^{20-22}$ have shown that chemical interface damping induced by adsorbate molecules increases the homogeneous spectral linewidth of a single plasmonic nanoparticle. One could argue that if every single nanoparticle within an ensemble displays the same amount of spectral redshift, the ensemble spectrum should broaden as a summation of individual damping. However, a decrease in the ensemble spectral linewidth is consistently observed for plasmonic nanoparticles, upon both SAM formation and analyte binding 
(see Table S1 for the estimated peak widths). ${ }^{13,23-27}$ The reason for such narrowing phenomenon has never been discussed.

Herein, an ensemble hypothesis along with experimental evidence is presented as an interpretation of the ensemble narrowing effect, based on which a novel avenue of sensing is proposed using the classic biotin-streptavidin system as a demonstration. This portable sensing platform is highly adaptable and allows the simultaneous register of both LSPR peak position and bandwidth, providing comprehensive spectral information as well as possibilities of extrinsic sensitivity enhancement, real-time measurement and facile miniaturization.

\section{EXPERIMENTAL SECTION}

2.1. Materials. Chloro(trimethylphosphine)gold(I) (99\%), methyl lithium (1.6 M in diethyl ether), lithium aluminum hydride (95\%), magnesium sulfate, chlorobenzene, trichloro(octadecyl)silane (OTS), and 11-mercapto-1-undecanol (97\%; referred to as alkane thiol hereafter) were acquired from Sigma Aldrich (Ontario, Canada). Sulfuric acid (98\%), hydrogen peroxide (30\%), acetone, ethanol, methanol, 1-propanol, and toluene were obtained from Caledon Laboratories Ltd. (Ontario, Canada). Polystyrene granules and polymethylmethacrylate (PMMA) sheets were obtained from Goodfellow Cambridge Ltd. (Huntingdon, England). Monothiolalkane PEG-biotin was purchased from SensoPath Technologies (Montana, USA; referred to as biotinylated thiol hereafter). Streptavidin was obtained from Rocklan Immunochemicals Inc. (Pennsylvania, USA). Plain microscope slides were purchased from Bio Nuclear Diagnostics Inc. (Ontario, Canada). Diethyl ether was distilled from lithium aluminum hydride and degassed before use. Water used during this study was deionized, obtained from Millipore cartridges. All other 
materials were used without further purification. Organometallic chemical vapor deposition (OMCVD) was carried out in a custom-made glass chamber.

2.2. OMCVD. Methyl(trimethylphosphine)gold(I) was synthesized as previously described in the literature. ${ }^{28}$ Gold nanoparticles (AuNPs) were deposited as previously described with modifications. ${ }^{28}$ Microscope slides were cut into $0.8 \mathrm{~cm}$ by $2.5 \mathrm{~cm}$ pieces and cleaned in $1: 1$ ethanol and acetone mixture by sonication for $30 \mathrm{~min}$ before use. Polymer substrate was prepared by spin coating polystyrene solution ( $1.5 \mathrm{~g}$ in $13 \mathrm{~mL}$ of chlorobenzene) on pre-cut PMMA $(0.8 \mathrm{~cm}$ by $2.5 \mathrm{~cm}$ ) at $2200 \mathrm{rpm}$. Hydrogen peroxide $(1 \mathrm{~mL})$ was added into UV ozone chamber to enhance the creation of surface $-\mathrm{OH}$ groups on microscope slides or PMMA substrates. OMCVD was carried out at $65.5^{\circ} \mathrm{C}$ under $13 \mathrm{~Pa}$ for 6-8 min to yield various sizes of AuNPs.

2.3. Scanning electron microscopy (SEM) characterization of AuNPs. The diameter range was determined with an SEM (Leo $1540 \mathrm{XB}$, Carl Zeiss, Oberkochen, Germany) under $20 \mathrm{kV}$ beam in back-scatter mode. $15 \mathrm{~nm}$ of aluminum was sputtered onto substrates prior to measurements. The microscopy images are of low quality due to the non-conducting nature of glass. Four different samples with LSPR peak wavelength of $532.5 \mathrm{~nm}, 578.0 \mathrm{~nm}, 552.0 \mathrm{~nm}$ and $514.5 \mathrm{~nm}$ were measured. Their respective diameters were $6.6 \pm 1.2 \mathrm{~nm}$ (Fig. S1 A), $8.6 \pm 1.9 \mathrm{~nm}$ (Fig. S1B), $7.4 \pm 1.6 \mathrm{~nm}$ (Fig. S1C), and $5.6 \pm 1.3 \mathrm{~nm}$ (Fig. S1D). Sample B was not used for spectral study due to the crowdedness of the nanoparticles. Its size measurement represents the upper limit of the nanoparticles studied. Since the mean diameter increases with LSPR wavelength ${ }^{29}$, nanoparticles from all the samples studied fall within the regime of electrostatic approximation. $^{30}$

2.4. Biosensing. All absorption spectra were obtained in water for glass substrates and in air for polymer substrates, using a Lambda 850 UV-vis spectrometer (Perkin Elmer, USA) in 
transmission mode. The scanning was performed between 400 and $800 \mathrm{~nm}$, in increments of 0.5 $\mathrm{nm}$ for glass substrates and $2 \mathrm{~nm}$ for polymer substrates. The SAM was prepared with 95:5 (molar ratio) of alkane thiol to biotinylated thiol in a total concentration of $5 \times 10^{-4} \mathrm{M}$ ethanolic solution. AuNP samples were functionalized with an excess of thiol solution for 18 24 h. Each sample was exposed to higher and higher concentration of aqueous streptavidin solutions between $10^{-12}$ and $10^{-5} \mathrm{M}$. Each concentration was allowed at least $3 \mathrm{~h}$ reaction time. The samples were rinsed thoroughly with water before taking their spectra to minimize unspecific binding. Bulk sensing was performed in the same configuration.

2.5. Calculation of ensemble linewidth (ELW). The ensemble linewidth, ELW, is defined as the spectral distance between the LSPR peak position $\left(\lambda_{0}\right)$ and the half-maximum position $\left(\lambda_{1}\right)$ toward the direction of longer wavelengths, such as the red and the blue arrows in Fig. 1. All spectra were corrected for baseline and normalized before ELW was extracted. The baseline was set to be the absorption value at $700 \mathrm{~nm}$ from each raw spectrum due to the presence of strong interference pattern from the substrate between 700 and $800 \mathrm{~nm}$. The normalization was performed so that the maximum absorption is unity. The LSPR peak position $\left(\lambda_{0}\right)$ was the spectral position with the highest absorption value. The half-maximum position $\left(\lambda_{1}\right)$ was found from actual spectra using an iterative process to select the spectral position whose absorption is closest to 0.5 . The above steps were executed using MATLAB 2016. 


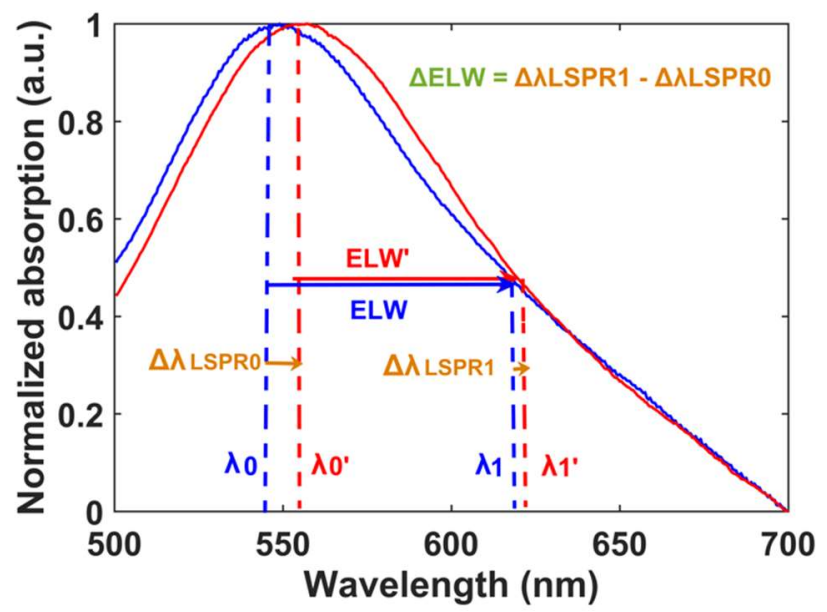

Figure 1. The normalized and baseline corrected absorption spectrum of an ensemble of AuNPs before (blue) and after (red) being functionalized with the mixed thiol SAM. The shift of maximum position $\left(\lambda_{0} \rightarrow \lambda_{0}{ }^{\prime}\right)$ and half maximum position $\left(\lambda_{1} \rightarrow \lambda_{1}{ }^{\prime}\right)$ determines the values of $\Delta \lambda_{\text {LSPR } 0}$ and

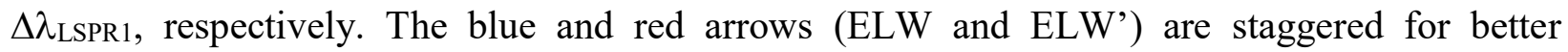
visualization.

\section{RESULTS AND DISCUSSION}

3.1. Premises of the ensemble hypothesis and their validity. As defined in section 2.5 , ELW excludes the spectral region on the low-energy side of the resonance peak, so that interband transition near the L-symmetry point with onset energy of $2.4 \mathrm{eV}$ is avoided for its effect on the spectral linewidth of single nanoparticles, thus also on the ELW. ${ }^{31}$ Although there is another interband transition near the X-symmetry point with onset energy of $1.8 \mathrm{eV}$, its absorption cross section is much weaker in comparison to the L-symmetry transition. ${ }^{32-34}$ Thus, the X-symmetry interband transition has negligible contribution to the ELW. This argument can also be supported experimentally, where surface-immobilized AuNPs were exposed to two different bulk refractive 
indices (Fig. 2A). The ELW stayed constant within measurement uncertainty even though the reversible shift of the plasmon peak altered the spectral overlap between the X-symmetry transition edge and the LSPR peak position.
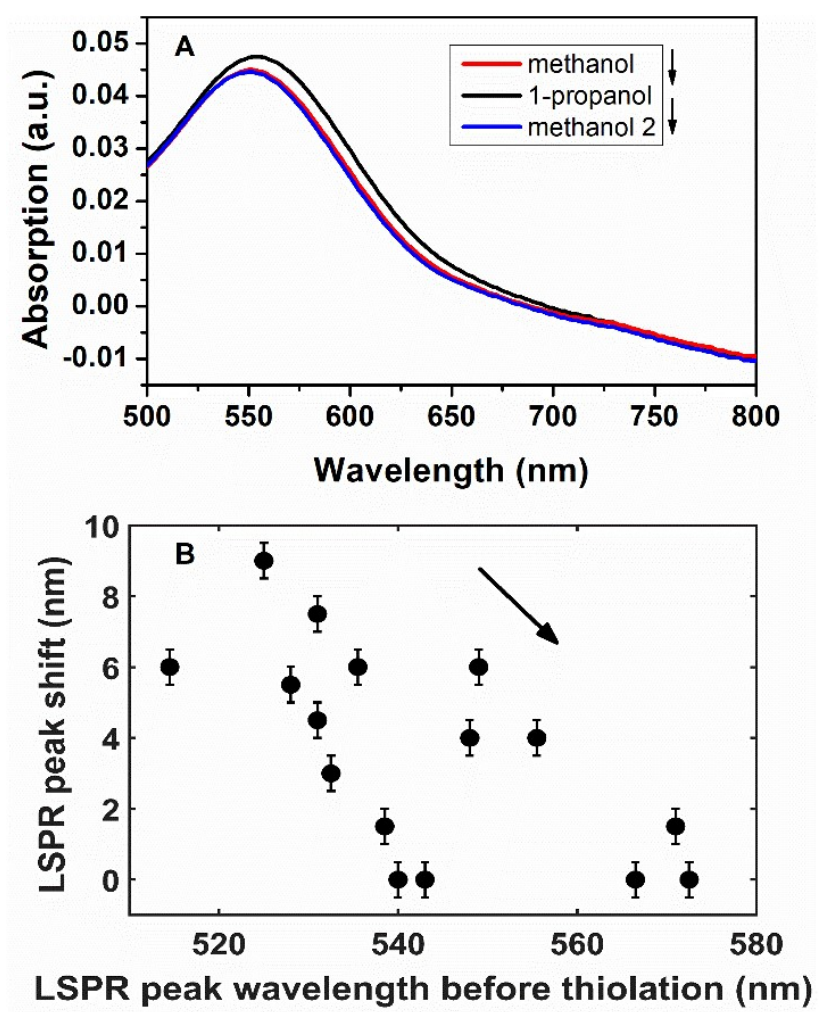

Figure 2. (A) The raw absorption spectra of surface-immobilized AuNPs exposed to methanol (n $=1.33)$, 1-propanol $(\mathrm{n}=1.38)$, then back to methanol. ELW $=48 \pm 2 \mathrm{~nm}$ for all three peaks. (B) The redshift of LSPR peak position as a result of thiolation versus the LSPR peak wavelength before the formation of thiol SAM. Each data point represents a different ensemble/sample. The trend is highlighted with an arrow.

The four premises of the ensemble hypothesis are: (1) AuNPs are immobilized on a substrate; (2) there is a size distribution within an ensemble of AuNPs so that the resonance peak position $\left(\lambda_{0}\right)$ is controlled by the predominant size and that the half maximum position $\left(\lambda_{1}\right)$ is determined 
by larger particles; (3) all AuNPs have similar bulk sensitivity; (4) the ad-layer does not extend far beyond the evanescent decay length of AuNPs.

The first premise is based on the fact that SAM molecules displace the surface anions of chemically reduced colloids, disrupting the repulsive forces that stabilize the suspension. Thus, the observed optical changes for colloidal plasmonic nanoparticles should largely be attributed to the aggregation of colloids and not to the presence of thiol capping layers. ${ }^{35-37}$ The ensemble hypothesis is therefore only valid for surface immobilized nanoparticles. In fact, ensemble peak broadening instead of narrowing has been observed for colloidal plasmonic nanoparticles. ${ }^{14,38-39}$

The second premise should be valid for a realistic ensemble with a reasonable size distribution as long as the LSPR peak position redshifts as the particle size increases (see Fig. S1). ${ }^{29-31}$ The ensemble spectrum can therefore be described as the sum of single particle spectra multiplied by their respective weighting factors according to the size distribution. ${ }^{40}$

The third premise is supported theoretically ${ }^{41}$ as all the AuNPs studied fall within the regime of electrostatic approximation (see section 2.3 and Fig. S1 for size measurements). It is also corroborated by the bulk sensing experiment (Fig. 2A) in that all the AuNPs exhibited the same amount of redshift and blueshift reversibly, leading to a constant ELW throughout a cycle of change in bulk refractive index. Moreover, it has been shown explicitly that the bulk sensitivity of surface immobilized AuNPs fall within a narrow range regardless of the size for particles up to 48 $\mathrm{nm}$ in diameter. ${ }^{31}$ The fourth premise should generally hold true for small organic molecules. Otherwise, the exponentially decaying field will lead to a weak response. Since the decay length is comparable to the particle size, an ad-layer of around $10 \mathrm{~nm}$ in thickness is reasonable for simple systems such as biotin-streptavidin, whose swollen thickness is around $6 \mathrm{~nm} .{ }^{42}$ 
3.2. Ensemble hypothesis. For an ensemble of AuNPs, let the diameter and the evanescent decay length of the predominant particle size (peaking at $\lambda_{0}$ ) denoted by $\mathrm{D}_{0}$ and $\mathrm{I}_{0}$; and those of the particles peaking at $\lambda_{1}$ by $D_{1}$ and $I_{1}$. Quantitatively, the shift of the $\lambda_{0}$ position of an ensemble spectrum $\left(\Delta \lambda_{\text {LSPR0 }}\right.$, equation 1$)$ due to a single adsorbed layer of uniform thickness can be expressed in terms of its refractive index $\left(n_{a}\right)$ and thickness $(\Delta d)$; refractive index of the solvent $\left(\mathrm{n}_{\mathrm{s}}\right)$; characteristic decay length $\left(\mathrm{I}_{0}\right)$ and bulk sensitivity $(\mathrm{m})$ of the nanoparticles. ${ }^{43}$ The shift of the $\lambda_{1}$ position ( $\left.\Delta \lambda_{\text {LSPR } 1}\right)$ can be obtained the same way by substituting $I_{1}$ for $I_{0}$ and $\lambda_{1}$ for $\lambda_{0}$. Therefore, the change in ensemble linewidth ( $\triangle E L W$, equation 2$)$ can be expressed as the difference between $\Delta \lambda_{\text {LSPR } 1}$ and $\Delta \lambda_{\text {LSPR } 0}$ as illustrated in Fig. 1.

$$
\begin{gathered}
\lambda_{0}^{\prime}-\lambda_{0}=\Delta \lambda_{L S P R 0}=m\left(n_{a}-n_{S}\right)\left(1-e^{-\frac{2 \Delta d}{I_{0}}}\right) \\
\Delta E L W=\Delta \lambda_{L S P R}-\Delta \lambda_{L S P R}=m\left(n_{a}-n_{S}\right)\left(e^{-\frac{2 \Delta d}{I_{0}}}-e^{-\frac{2 \Delta d}{I_{1}}}\right)
\end{gathered}
$$

Given that $\mathrm{m},\left(\mathrm{n}_{\mathrm{a}}-\mathrm{n}_{\mathrm{S}}\right)$, and $\Delta \mathrm{d}$ terms are the same for all sizes within an ensemble, $\Delta \lambda_{\mathrm{LSPR} 0}$ and $\triangle E L W$ are entirely determined by the exponential terms in both equations. Since smaller nanoparticles have shorter decay lengths ${ }^{44}$, their $1-e^{-\frac{2 \Delta d}{I_{0}}}$ terms are larger, leading to more pronounced shifts. In fact, smaller particles are more sensitive due to a more confined electromagnetic field and a larger analyte to sensing volume ratio. ${ }^{3,45-46}$ This comparison is supported both theoretically ${ }^{41}$ and experimentally (Fig. 2B) where smaller AuNPs peaking at shorter LSPR wavelengths exhibited larger redshifts after the formation of thiol SAM. Since the trend of smaller AuNPs exhibiting higher surface sensitivity is valid for particles up to $80 \mathrm{~nm}$ in diameter and an ad-layer of $15 \mathrm{~nm}$ in thickness ${ }^{41}$, the ensemble hypothesis should be applicable in a wide range of situations as long as the four premises are satisfied. This allows the interpretation 
of ensemble narrowing observed in the literature ${ }^{13,23-27}$ and ensures the adaptability of the sensing platform we will propose in section 3.3.
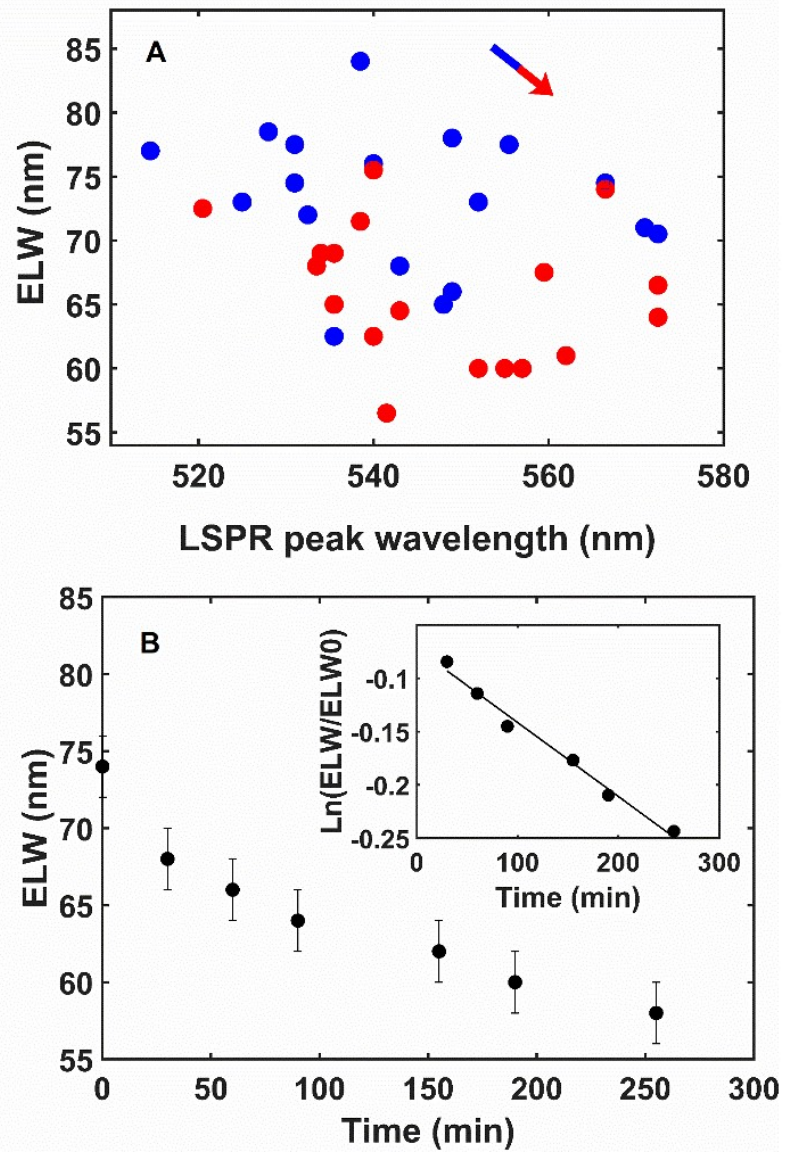

Figure 3. (A) ELWs of different AuNP ensembles before (blue) and after (red) being functionalized with the mixed thiol SAM. The trend is highlighted with an arrow. Each blue point or each red point represents a different ensemble/sample. See Table S2 for their numerical values. The error bars are within the size of the symbols. (B) Time evolution of the ELW for one ensemble of AuNPs during the formation of the mixed thiol SAM. The inset shows a first order kinetic for the ELW with respect to time. All spectra were taken in water. 
Within an ensemble of AuNPs, since $\mathrm{D}_{0}$ is smaller than $\mathrm{D}_{1}, \Delta \lambda_{\mathrm{LSPR} 0}$ is larger than $\Delta \lambda_{\mathrm{LSPR} 1}$, the ELW decreases as a result of thiolation. Indeed, the decrease in ELWs for 18 arbitrarily chosen ensembles after being functionalized with the thiol mixture (Fig. 3A) confirms the above conclusion and the narrowing effect observed in the literature. ${ }^{13,23-27}$ In short, the ELW is decreased due to a differential behavior that arises from the ensemble size distribution: smaller particles corresponding to the blue side of a spectrum display larger redshifts than the larger ones that contribute to the red side of the spectrum.

The time progression of ELW during SAM formation reveals a first order kinetic plot (Fig. 3B) even though the plasmon peak position $\left(\lambda_{0}\right)$ remains unchanged (see Fig. S2 for raw spectra). Thus, ELW unveils information that might otherwise be lost by monitoring the resonance peak position alone.

3.3. ELW for biosensing. The continuation of ensemble narrowing from SAM formation to analyte binding is observed for AuNPs immobilized on both glass and polystyrene substrates (Fig. 4A and 4B respectively, green triangles). The shift of LSPR peak wavelength (Fig. 4A and 4B, orange circles), on the other hand, increases with a growing thickness and refractive index of the biotin-streptavidin layer. Because there will always be some degree of surface defects in SAM layers over the probing area of the UV-vis spectrometer, as can be confirmed by the broad distribution of shift response in Fig. 2B, the bound streptavidin and the biotinylated SAM are regarded as one single ad-layer. In fact, treating two imperfect layers as separate entities distorts the estimation of $\mathrm{n}_{\mathrm{a}} \cdot{ }^{42}$ Therefore equation 1 and 2 are still valid, with $\Delta \lambda_{\mathrm{LSPR} 0}$ and $\Delta \mathrm{ELW}$ describing the shift of LSPR peak wavelength and the change in ensemble linewidth with respect to bare AuNPs in an aqueous environment. 

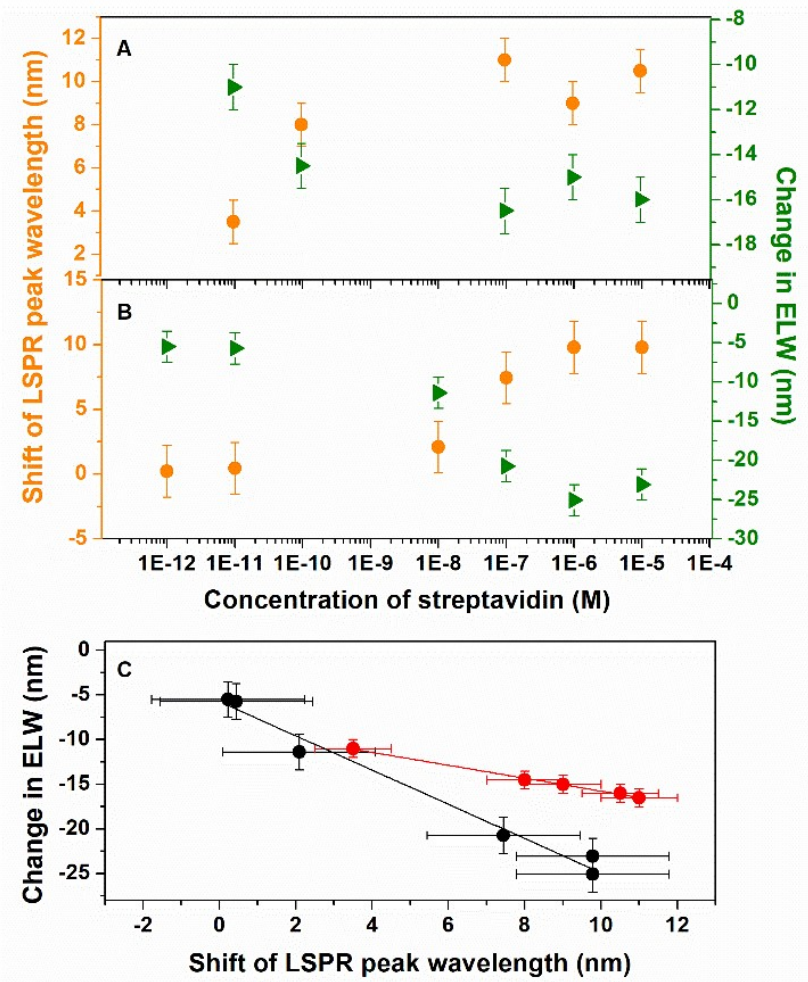

Figure 4. LSPR peak shift (orange circles) and change in ELW (green triangles) with respect to bare AuNPs versus concentration of streptavidin for (A) a glass substrate in water and (B) a polystyrene substrate in air. (C) Linear correlation between the change in ELW and the shift of LSPR peak wavelength. The slope and $\mathrm{R}^{2}$ are -0.72 and 0.99 for the glass substrate (red); -1.9 and 0.98 for the polystyrene substrate (black).

As more streptavidin molecules bind to biotin, $\Delta d$ and $\left(n_{a}-n_{s}\right)$ increase as functions of streptavidin concentration. Again, the $m, \Delta d$ and $\left(n_{a}-n_{s}\right)$ terms are the same for all the nanoparticles within a population. Plotting $e^{-\frac{2 \Delta d}{I_{0}}}-e^{-\frac{2 \Delta d}{I_{1}}}$ versus $1-e^{-\frac{2 \Delta d}{I_{0}}}$ gives a quasi-linear correlation between $\triangle \mathrm{ELW}$ and $\Delta \mathrm{LSPR}_{0}$, as long as $\Delta \mathrm{d}$ is still comparable to $\mathrm{I}_{0}$ (Fig. S3). The predicted linearity is corroborated for AuNPs on both glass and polystyrene substrates (Fig. 4C). Furthermore, the theoretical slope of this quasi-linear relationship becomes steeper with an 
increasing difference between $\mathrm{I}_{0}$ and $\mathrm{I}_{1}$ (Fig. S3). Since a broad size distribution (large $\mathrm{I}_{1}-\mathrm{I}_{0}$ ) entails a large resonance linewidth that decreases the figure of merit (FOM) of plasmonic nanoparticles, ${ }^{47}$ monitoring the change in ELW instead of wavelength shift proves more advantageous when the slope is steeper than negative one; where the slope is determined by the values of $\Delta d, I_{0}$ and $I_{1}$. The linearity between ELW and LSPR peak position urges for a new sensing avenue.

Rather than a stand-alone sensing pathway, registering both ELW and LSPR peak wavelength imparts comprehensiveness, flexibility and portability. This can be achieved with a simplified transmission-based platform consisting of a prism and a photodiode array (TOC Figure) that disperses and detects the transmitted white light sequentially. Without any moving component, this configuration can be miniaturized more readily. ELW encodes more spectral information than LSPR wavelength alone; but the latter requires less computational effort and memory space, because ELW is calculated based on the pixel distance between $\lambda_{0}$ and $\lambda_{1}$ rather than the pixel position of $\lambda_{0}$ alone. Users should be able to choose either or both of the parameters depending on their needs. 


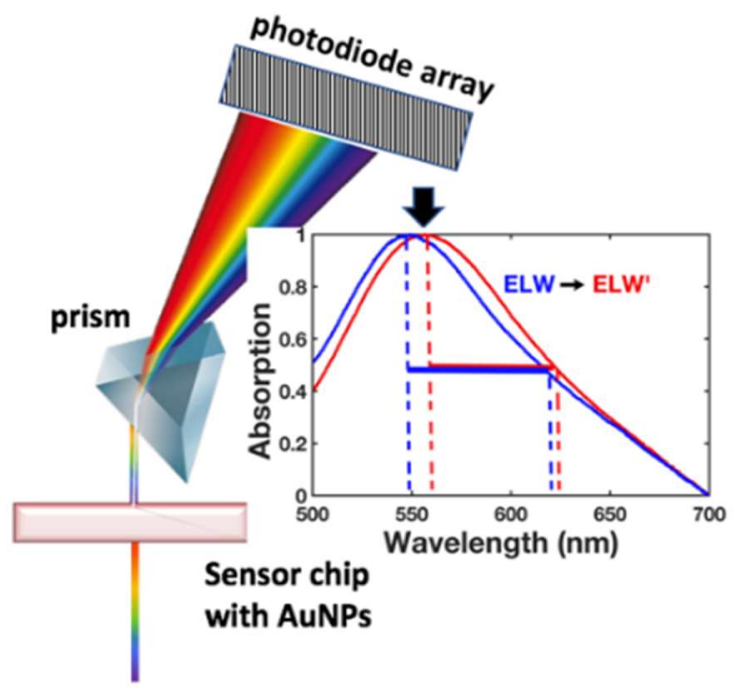

Figure 5. Proposed sensing platform without moving components based on the ensemble narrowing effect. A white light beam is incident on the sample carrying the AuNP. The transmitted light is dispersed by a prism and collected by a diode array delivering both ELW and spectral peak position.

Since ELW is calculated as the pixel distance between $\lambda_{0}$ and $\lambda_{1}$, the measurement uncertainty is double of what would be for reading the peak shift alone. However, $\mathrm{S} / \mathrm{N}$ can be enhanced by smoothing algorithm such as peak centroid fitting if necessary. ${ }^{48}$ Curvature has been proved useful for monitoring spectral changes without the influence of zeroth and first order noise such as drift and tilt. ${ }^{13}$ However, taking second derivative of absorption with respect to wavelength needs to be performed graphically, thus is not suitable for a portable device with real-time readout. These types of noise can be minimized by integrated a series of algorithms: Fourier transform, digital filters, and then an inverse Fourier transform. The methods stated here are all extrinsic methods that can 
be applied in conjunction with other intrinsic improvements outlined in the Introduction to further enhance the sensitivity and $\mathrm{S} / \mathrm{N}$.

\section{CONCLUSIONS}

The narrowing of ELW observed in the literature ${ }^{13,23-27}$ can be explained by a differential shifting behavior that arises from the ensemble size distribution as the thickness of adsorbed layers increases. This ensemble hypothesis is corroborated by the experimental evidence presented herein. While both are a function of analyte concentration, ELW reveals more spectral details than plasmon peak position, and can lead to a higher FOM if the slope of $\Delta$ ELW versus $\Delta \mathrm{LSPR}_{0}$ is steeper than negative one. A portable transmission-based sensing platform (TOC) allowing the simultaneous measurement of both LSPR wavelength and ELW is proposed. In comparison to conventional sensing configurations, the proposed platform not only reveals more spectral details and permits real-time measurements, but also present a possibility of sensitivity enhancement and miniaturization. This platform can also be adapted for other types of plasmonic nanoaprticles with different size ranges and geometries as long as the four premises of the ensemble hypothesis are satisfied.

\section{ASSOCIATED CONTENT}

\section{Supporting Information.}

The Supporting Information is available free of charge on the ACS Publications website at DOI.

Table S1 and S2; Fig. S1, S2 and S3 (PDF). 


\section{AUTHOR INFORMATION}

\section{Corresponding Author}

*(S.M.).smittler@uwo.ca

\section{ACKNOWLEDGMENT}

Z.D. and S.M. are grateful to the Natural Sciences and Engineering Research Council of Canada (NSERC) for fund RGPIN 2014-05793. The Western Nanofabrication Facility is thanked for the availability of the UV Ozone generator. The authors would also like to thank Todd Simpson from the Western Nanofabrication Facility for taking the electron microscopy images.

\section{Notes}

The authors declare no competing financial interests.

\section{REFERENCES}

1. Link, S.; El-Sayed, M. A. Size and Temperature Dependence of the Plasmon Absorption of Colloidal Gold Nanoparticles. J. Phys. Chem. B 1999, 103 (21), 4212-4217.

2. Link, S.; El-Sayed, M. A. Shape and size dependence of radiative, non-radiative and photothermal properties of gold nanocrystals. Int. Rev. Phys. Chem. 2000, 19 (3), 409-453.

3. Anker, J. N.; Hall, W. P.; Lyandres, O.; Shah, N. C.; Zhao, J.; Van Duyne, R. P. Biosensing with plasmonic nanosensors. Nat. Mater. 2008, 7 (6), 442-453. 
4. Willets, K. A.; Van Duyne, R. P. Localized Surface Plasmon Resonance Spectroscopy and Sensing. Annu. Rev. Phys. Chem. 2007, 58 (1), 267-297.

5. Johansen, K.; Stalberg, R.; Lundström, I.; Liedberg, B. Surface plasmon resonance: instrumental resolution using photo diode arrays. Meas. Sci. Technol. 2000, 11 (11), 1630.

6. Johansen, K.; Lundström, I.; Liedberg, B. Sensitivity deviation: instrumental linearity errors that influence concentration analyses and kinetic evaluation of biomolecular interactions. Biosens. Bioelectron. 2000, 15 (9), 503-509.

7. Shiohara, A.; Novikov, S. M.; Solís, D. M.; Taboada, J. M.; Obelleiro, F.; Liz-Marzán, L. M. Plasmon Modes and Hot Spots in Gold Nanostar-Satellite Clusters. J. Phys. Chem. C 2015, $119(20), 10836-10843$.

8. Lee, T. K.; Kwak, S. K. Effects of LSPR of Gold Nanospheres by Surface Vacancies and Protruding Tips. J. Phys. Chem. C 2014, 118 (11), 5881-5888.

9. Shen, Y.; Zhou, J.; Liu, T.; Tao, Y.; Jiang, R.; Liu, M.; Xiao, G.; Zhu, J.; Zhou, Z.-K.; Wang, X. et al. Plasmonic gold mushroom arrays with refractive index sensing figures of merit approaching the theoretical limit. Nat. Commun. 2013, 4, 2381.

10. King, N. S.; Liu, L.; Yang, X.; Cerjan, B.; Everitt, H. O.; Nordlander, P.; Halas, N. J. Fano Resonant Aluminum Nanoclusters for Plasmonic Colorimetric Sensing. ACS Nano 2015, 9 (11), 10628-10636. 
11. Nugroho, F. A. A.; Iandolo, B.; Wagner, J. B.; Langhammer, C. Bottom-Up Nanofabrication of Supported Noble Metal Alloy Nanoparticle Arrays for Plasmonics. ACS Nano 2016, 10 (2), 2871-2879.

12. Ferhan, A. R.; Kim, D.-H. Nanoparticle polymer composites on solid substrates for plasmonic sensing applications. Nano Today 2016, 11 (4), 415-434.

13. Chen, P.; Liedberg, B. Curvature of the Localized Surface Plasmon Resonance Peak. Anal. Chem. 2014, 86 (15), 7399-7405.

14. Chen, P.; Tran, N. T.; Wen, X.; Xiong, Q.; Liedberg, B. Inflection Point of the Localized Surface Plasmon Resonance Peak: A General Method to Improve the Sensitivity. ACS Sensors 2017, 2 (2), 235-242.

15. Skoog, D. A.; Holler, F. J.; Crouch, S. R. Principles of Instrumental Analysis, 6th ed.; Brooks Cole, 2006.

16. Piscevic, D.; Knoll, W.; Tarlov, M. J. Surface plasmon microscopy of biotin-streptavidin binding reactions on UV-photopatterned alkanethiol self-assembled monolayers. Supramol. Sci. 1995, 2 (2), 99-106.

17. Spinke, J.; Liley, M.; Schmitt, F. J.; Guder, H. J.; Angermaier, L.; Knoll, W. Molecular recognition at self - assembled monolayers: Optimization of surface functionalization. J. Chem. Phys. 1993, 99 (9), 7012-7019. 
18. Zijlstra, P.; Paulo, P. M. R.; Yu, K.; Xu, Q.-H.; Orrit, M. Chemical Interface Damping in Single Gold Nanorods and Its Near Elimination by Tip-Specific Functionalization. Angew. Chem. Int. Ed. 2012, 51 (33), 8352-8355.

19. Foerster, B.; Joplin, A.; Kaefer, K.; Celiksoy, S.; Link, S.; Sönnichsen, C. Chemical Interface Damping Depends on Electrons Reaching the Surface. ACS Nano 2017, 11 (3), 28862893.

20. Kreibig, U. Interface-induced dephasing of Mie plasmon polaritons. Appl. Phys. B 2008, $93(1), 79-89$.

21. Douglas-Gallardo, O. A.; Berdakin, M.; Sánchez, C. G. Atomistic Insights into Chemical Interface Damping of Surface Plasmon Excitations in Silver Nanoclusters. J. Phys. Chem. C 2016, 120 (42), 24389-24399.

22. Hövel, H.; Fritz, S.; Hilger, A.; Kreibig, U.; Vollmer, M. Width of cluster plasmon resonances: Bulk dielectric functions and chemical interface damping. Phys. Rev. B 1993, 48 (24), 18178-18188.

23. Hall, W. P.; Ngatia, S. N.; Van Duyne, R. P. LSPR Biosensor Signal Enhancement Using Nanoparticle-Antibody Conjugates. J. Phys. Chem. C 2011, 115 (5), 1410-1414.

24. Oh, S. Y.; Heo, N. S.; Shukla, S.; Cho, H.-J.; Vilian, A. T. E.; Kim, J.; Lee, S. Y.; Han, Y.K.; Yoo, S. M.; Huh, Y. S. Development of gold nanoparticle-aptamer-based LSPR sensing chips for the rapid detection of Salmonella typhimurium in pork meat. Sci. Rep. 2017, 7 (1), 10130. 
25. Barbillon, G. Plasmonic Nanostructures Prepared by Soft UV Nanoimprint Lithography and Their Application in Biological Sensing. Micromachines 2012, 3 (1), 21-27.

26. Yoo, S. Y.; Kim, D.-K.; Park, T. J.; Kim, E. K.; Tamiya, E.; Lee, S. Y. Detection of the Most Common Corneal Dystrophies Caused by BIGH3 Gene Point Mutations Using a Multispot Gold-Capped Nanoparticle Array Chip. Anal. Chem. 2010, 82 (4), 1349-1357.

27. Endo, T.; Kerman, K.; Nagatani, N.; Takamura, Y.; Tamiya, E. Label-Free Detection of Peptide Nucleic Acid-DNA Hybridization Using Localized Surface Plasmon Resonance Based Optical Biosensor. Anal. Chem. 2005, 77 (21), 6976-6984.

28. Kandeepan, S.; Paquette, J. A.; Gilroy, J. B.; Mittler, S. OMCVD Gold Nanoparticles Covalently Attached to Polystyrene for Biosensing Applications. Chem. Vap. Deposition 2015, 21 (10-11-12), 275-280.

29. Ertorer, E. Fabricating Cost-Effective Nanostructures for Biomedical Applications. Ph.D. Dissertation, The University of Western Ontario, London, ON, 2013.

30. Jensen, T.; Kelly, L.; Lazarides, A.; Schatz, G. C. Electrodynamics of Noble Metal Nanoparticles and Nanoparticle Clusters. J. Cluster Sci. 1999, 10 (2), 295-317.

31. Nath, N.; Chilkoti, A. Label-Free Biosensing by Surface Plasmon Resonance of Nanoparticles on Glass: Optimization of Nanoparticle Size. Anal. Chem. 2004, 76 (18), 53705378.

32. Guerrisi, M.; Rosei, R.; Winsemius, P. Splitting of the interband absorption edge in Au. Phys. Rev. B 1975, 12 (2), 557-563. 
33. Ngoc, L. L. T.; Wiedemair, J.; van den Berg, A.; Carlen, E. T. Plasmon-modulated photoluminescence from gold nanostructures and its dependence on plasmon resonance, excitation energy, and band structure. Opt. Express 2015, 23 (5), 5547-5564.

34. Aspnes, D. E.; Kinsbron, E.; Bacon, D. D. Optical properties of Au: Sample effects. Phys. Rev. B 1980, 21 (8), 3290-3299.

35. Mirkin, C. A.; Letsinger, R. L.; Mucic, R. C.; Storhoff, J. J. A DNA-based method for rationally assembling nanoparticles into macroscopic materials. Nature 1996, 382 (6592), 607609.

36. Malinsky, M. D.; Kelly, K. L.; Schatz, G. C.; Van Duyne, R. P. Chain Length Dependence and Sensing Capabilities of the Localized Surface Plasmon Resonance of Silver Nanoparticles Chemically Modified with Alkanethiol Self-Assembled Monolayers. J. Am. Chem. Soc. 2001, 123 (7), 1471-1482.

37. Weisbecker, C. S.; Merritt, M. V.; Whitesides, G. M. Molecular Self-Assembly of Aliphatic Thiols on Gold Colloids. Langmuir 1996, 12 (16), 3763-3772.

38. Linnert, T.; Mulvaney, P.; Henglein, A. Surface chemistry of colloidal silver: surface plasmon damping by chemisorbed iodide, hydrosulfide (SH-), and phenylthiolate. J. Phys. Chem. 1993, 97 (3), 679-682.

39. Crespo, P.; Litrán, R.; Rojas, T. C.; Multigner, M.; de la Fuente, J. M.; Sánchez-López, J. C.; García, M. A.; Hernando, A.; Penadés, S.; Fernández, A. Permanent Magnetism, Magnetic Anisotropy, and Hysteresis of Thiol-Capped Gold Nanoparticles. Phys. Rev. Lett. 2004, 93 (8), 087204. 
40. Hu, Z. J.; Hou, S.; Ji, Y. L.; Wen, T.; Liu, W. Q.; Zhang, H.; Shi, X. W.; Yan, J.; Wu, X. C. Fast characterization of gold nanorods ensemble by correlating its structure with optical extinction spectral features. AIP Adv. 2014, 4 (11), 117137.

41. Kvasnička, P.; Homola, J. Optical sensors based on spectroscopy of localized surface plasmons on metallic nanoparticles: Sensitivity considerations. Biointerphases 2008, 3 (3), FD4FD11.

42. Mittler, S.; Menges, B. Evanescent Waves as Nanoprobes for Surfaces and Interfaces: From Waveguide Technology to Sensor Application. In: Frontiers in Surface Nanophotonics. Optical Sciences, vol 133. Springer New York: New York, NY, 2007.

43. Jung, L. S.; Campbell, C. T.; Chinowsky, T. M.; Mar, M. N.; Yee, S. S. Quantitative Interpretation of the Response of Surface Plasmon Resonance Sensors to Adsorbed Films. Langmuir 1998, 14 (19), 5636-5648.

44. Huang, X.; El-Sayed, M. A. Gold nanoparticles: Optical properties and implementations in cancer diagnosis and photothermal therapy. J. Adv. Res. 2010, 1 (1), 13-28.

45. Kedem, O.; Tesler, A. B.; Vaskevich, A.; Rubinstein, I. Sensitivity and Optimization of Localized Surface Plasmon Resonance Transducers. ACS Nano 2011, 5 (2), 748-760.

46. Beeram, S. R.; Zamborini, F. P. Effect of Protein Binding Coverage, Location, and Distance on the Localized Surface Plasmon Resonance Response of Purified Au Nanoplates Grown Directly on Surfaces. J. Phys. Chem. C 2011, 115 (15), 7364-7371. 
47. Mayer, K. M.; Hafner, J. H. Localized Surface Plasmon Resonance Sensors. Chem. Rev. 2011, $111(6), 3828-3857$.

48. Nusz, G. J.; Marinakos, S. M.; Curry, A. C.; Dahlin, A.; Höök, F.; Wax, A.; Chilkoti, A. Label-Free Plasmonic Detection of Biomolecular Binding by a Single Gold Nanorod. Anal. Chem. 2008, 80 (4), 984-989. 
